\title{
Multi-baseline coherence optimisation in partial and compact polarimetric modes
}

\author{
A. Reigber ${ }^{1}$, M. Neumann ${ }^{2,3}$, L. Ferro-Famil ${ }^{2}$, M. Jäger ${ }^{3}$ and P. Prats ${ }^{1}$ \\ ${ }^{1}$ German Aerospace Center (DLR), Microwaves and Radar Institute, Oberpfaffenhofen, Germany \\ P.O. Box 1116, D-82234 Weßling, Tel.: ++49-8153-282360, Email. andreas.reigber@dlr.de \\ ${ }^{2}$ University of Rennes 1, Institute of Electronics and Telecommunications of Rennes (IETR), Rennes, France \\ ${ }^{3}$ Berlin University of Technology (TUB), Computer Vision and Remote Sensing Group, Berlin, Germany
}

\begin{abstract}
Modern space-borne SAR sensors, like ALOSPALSAR, TerraSAR-X and Radarsat-2 all provide at least a "partial polarimetric mode", acquiring only 2 of the 4 elements of the Sinclair matrix, like for example $\mathrm{HH}$ and $\mathrm{HV}$ or VV and VH. In addition, it has been demonstrated that with certain so-called "compact PolSAR" single-transmit dual-receive techniques one can obtain an estimation of the fully polarimetric information. Such systems are attractive in terms of reduction of pulse repetition frequency, data rate, and complexity and are currently very popular. However, they do not acquire complete information pertaining to the full polarisation state of the target and, as a consequence also coherence optimisation suffers from the reduced configuration space.

In this paper, the potential of the different partial polarimetric setups for coherence optimisation is evaluated both theoretically and experimentally and compared to the capabilities of a fully polarimetric system. It will be analysed to which extent partial polarimetric system can improve the derivation of interferometric information from partly decorrelated surfaces, in particular of vegetated or even forested areas. Special attention is paid to the constrained coherence optimisation of multi-baseline setups, important for modern DInSAR techniques like PS analysis and continuous DInSAR monitoring in general. All experimental analyses will be performed using fully polarimetric multi-baseline data sets. For proper comparison, partial polarimetric information is derived from these by matrix transformations according to the respective transmit / receive configuration.
\end{abstract}

\section{INTRODUCTION}

In recent years, differential SAR interferometry has become an established technique for detecting and monitoring centimetre-scale deformations of the earths surface, as well as glacier flows and landslides [1]. Although often very efficient, differential SAR interferometry suffers from decorrelation effects, particularly when long data acquisition intervals are involved. SAR polarimetry is a technique, which offers the possibility of separating multiple scattering mechanisms inside the resolution cell. Polarimetric phase diversity allows the application of phase and coherence optimisation techniques in order to minimise temporal and spatial decorrelation effects. These capabilities are interesting for differential interferometry; in particular coherence optimisation can significantly improve the usability of long-term interferometric pairs [2].

Recently, several new 'compact' polarimetric imaging modes have been discussed in literature, which are signifi- cantly less demanding in terms of PRF, data rate and overall complexity than fully polarimetric data acquisitions [3] [4] [5]. It is promised that one can obtain an estimation of the fully polarimetric information by incorporating certain assumption on the scattering process. Obviously, the success of these compact modes depends on the applicability of these scattering models, i.e. on the target itself. With respect to DInSAR, it remains the question to which extent polarimetric coherence optimisation can be performed when instead of a fully polarimetric data acquisition only one of the limited compact polarimetric modes is available.

In this paper, the potential of the different partial polarimetric setups for coherence optimisation is evaluated both theoretically and experimentally and compared to the capabilities of a fully polarimetric system. The next section introduces in the theoretical background of full and partial polarimetric coherence optimisation. In section III, experimental results derived from DLR E-SAR data at L-band will be shown. They demonstrate that long-term DInSAR observations of vegetated surfaces are feasible and that coherence optimisation is a key technique for obtaining optimal results. Sec. IV will conclude the paper.

\section{Full and Partial Coherence Optimisation}

A multi-baseline $n$-track geometry contains $\frac{n}{2}(n-1)$ direct baselines. Fully polarimetric, monostatic data can be represented in the PAULI-basis, assuming reciprocity, by the scattering vector $\mathbf{k}_{i}$ in track $i \in[1, n]$ :

$$
\mathbf{k}_{i}=\frac{1}{\sqrt{2}}\left[S_{i}^{H H}+S_{i}^{V V}, S_{i}^{H H}-S_{i}^{V V}, 2 S_{i}^{H V}\right]^{T}
$$

The multi-baseline PolInSAR coherency matrix T, representing estimated covariance among the polarimetric and interferometric channels, is generated by multi-looking the outer product of the aggregated scattering vector $\mathbf{k}$ :

$$
\mathbf{T}=\left\langle\mathbf{k k}^{\dagger}\right\rangle=\left[\begin{array}{ccc}
\mathbf{T}_{11} & \ldots & \boldsymbol{\Omega}_{1 n} \\
\vdots & \ddots & \vdots \\
\boldsymbol{\Omega}_{1 n}^{\dagger} & \ldots & \mathbf{T}_{n n}
\end{array}\right], \quad \text { with } \mathbf{k}=\left[\begin{array}{c}
\mathbf{k}_{1} \\
\vdots \\
\mathbf{k}_{n}
\end{array}\right]
$$

where \langle\rangle denotes spatial averaging, and ${ }^{\dagger}$ the adjoint operator. $\mathbf{T}_{i i}$ contain the polarimetric information, while $\boldsymbol{\Omega}_{i j}(i \neq j)$ contain baseline dependent polarimetric and interferometric 
information. The coherence between two channels, in possibly different polarisations $\boldsymbol{\omega}_{i} \neq \boldsymbol{\omega}_{j}$, is defined as

$$
\gamma_{i j\left(\boldsymbol{\omega}_{i}, \boldsymbol{\omega}_{j}\right)}=\frac{\boldsymbol{\omega}_{i}^{\dagger} \boldsymbol{\Omega}_{i j} \boldsymbol{\omega}_{j}}{\sqrt{\boldsymbol{\omega}_{i}^{\dagger} \mathbf{T}_{i i} \boldsymbol{\omega}_{i} \boldsymbol{\omega}_{j}^{\dagger} \mathbf{T}_{j j} \boldsymbol{\omega}_{j}}}
$$

By choosing $\boldsymbol{\omega}_{i}$ and $\boldsymbol{\omega}_{j}$, the value of the coherence $\gamma$ can be influenced and an optimisation of their absolute value can be performed [6]. In the multi-baseline case, as described above, the situation is slightly more complex, since one has to decide whether a joint optimisation of the entire data set (i.e. over all possible pairs), or an individual optimisation of single interferometric pairs has to be performed. However, for multibaseline DInSAR application only the former is reasonable, because otherwise no comparability of the different data sets is given. In addition one has to decide to allow different polarisation states at two ends of the baseline or not [7]: The most general multi-baseline / multiple scattering mechanisms (MSM) method assigns a distinct scattering mechanism to each data set. This approach allows one to optimise the coherence for scattering mechanisms that might have different polarimetric signatures in different datasets. The equal scattering mechanisms (ESM) method, on the other hand, enforces equal polarimetric signatures along all baselines $\left(\boldsymbol{\omega}_{i}=\boldsymbol{\omega}_{j} \forall i, j\right)$, and the application of this method is restricted to a single scattering mechanism for the dominant scatterer.

In case of compact polarimetric imaging modes, only two independent channels are received by the sensor, causing a $2 \times 2$ PolSAR coherency matrix. In context of coherence optimisation, the reconstruction of the full $3 \times 3$ coherency matrix makes no sense: The two-dimensional observation space, in which the optimisation process is performed, would only be artifically extended to 3D. Any gain achieved by the reconstruction can only be caused by statistical sideeffects. In addition, the reconstruction of the multi-baseline PolInSAR coherency matrix $\mathbf{T}$ of Eq. 2 requires an estimation of the matrix elements $\Omega_{i j}$, which carry both polarimetric and interferometric information. This goes far beyond the PolSAR reconstruction techniques presented in [3]-[5] and is generally very questionable without precise knowledge about the target itself.

Therefore, in this paper compact PolSAR modes are treated as dual-channel modes and coherence optimisation is performed directly in the reduced $2 \mathrm{n} \times 2 \mathrm{n}$ observation space. Mathematically, this is equivalent to a degradation of the numerical range of the complex coherence [?], [7] to an ellipse in the complex unitary circle. This imposes further constraints into the optimisation process and principally causes maximum coherences, which are lower (or equal) to the ones of the fully polarimetric optimised case. However, the observation spaces of the different compact modes are not identical, and, depending on the target, coherence optimisation might be more or less successful. In the next chapter, these differences will be analysed experimentally.
TABLE I

ORIGINAL AND OPTIMISED COHERENCE VALUES FOR FULLY POLARIMETRIC MODES.

\begin{tabular}{|cc||ccc|cc|} 
ROI & Pair & HH & VV & XX & MSM & ESM \\
\hline \hline \multirow{4}{*}{ runway } & $1-2$ & 0,820 & 0,898 & 0,704 & 0,906 & 0,893 \\
& $1-3$ & 0,369 & 0,550 & 0,198 & 0,582 & 0,562 \\
& $1-4$ & 0,294 & 0,417 & 0,141 & 0,464 & 0,444 \\
& $3-4$ & 0,408 & 0,563 & 0,227 & 0,595 & 0,584 \\
\hline \multirow{3}{*}{ urban } & $1-2$ & 0,673 & 0,682 & 0,624 & 0,753 & 0,711 \\
& $1-3$ & 0,383 & 0,368 & 0,296 & 0,532 & 0,475 \\
& $1-4$ & 0,377 & 0,375 & 0,310 & 0,529 & 0,483 \\
& $3-4$ & 0,472 & 0,469 & 0,391 & 0,582 & 0,552 \\
\hline \multirow{3}{*}{ forest 1} & $1-2$ & 0,339 & 0,328 & 0,304 & 0,402 & 0,366 \\
& $1-3$ & 0,170 & 0,137 & 0,130 & 0,248 & 0,212 \\
& $1-4$ & 0,171 & 0,139 & 0,123 & 0,243 & 0,219 \\
& $3-4$ & 0,265 & 0,236 & 0,224 & 0,327 & 0,310 \\
\hline \multirow{4}{*}{ forest 2 } & $1-2$ & 0,402 & 0,359 & 0,345 & 0,448 & 0,401 \\
& $1-3$ & 0,232 & 0,153 & 0,156 & 0,300 & 0,265 \\
& $1-4$ & 0,199 & 0,145 & 0,141 & 0,271 & 0,247 \\
& $3-4$ & 0,330 & 0,262 & 0,259 & 0,384 & 0,367 \\
\hline \multirow{6}{*}{ low forest } & $1-2$ & 0,849 & 0,809 & 0,797 & 0,855 & 0,834 \\
& $1-3$ & 0,319 & 0,229 & 0,207 & 0,370 & 0,345 \\
& $1-4$ & 0,327 & 0,240 & 0,219 & 0,377 & 0,359 \\
& $3-4$ & 0,503 & 0,439 & 0,418 & 0,521 & 0,516 \\
\hline
\end{tabular}

\section{EXPERIMENTAL RESULTS}

For the experimental analysis, a stack of 4 fully polarimetric images of the test-site Oberpfaffenhofen / Germany has been used. The data have been acquired by DLR's E-SAR sensor at L-band over a time span of approximately one year: track 1 and 2 both were recorded August 27th 2001 (12min temporal baseline), Track 3 June 15th 2000 and Track 4 September 15th 2000. The interferometric pairs 1-3 and 1-4 posses a temporal baseline of about 1 year, while the pair 3-4 covers only 92 days. All spatial baselines are small, which allows to neglect volume decorrelation effects.

Fig. 2 shows the coherence map of the 3-4 pair in $\mathrm{HH}$, $\mathrm{VV}$ and $\mathrm{XX}$ polarisation, as well as the fully polarimetrically optimised coherence map (MSM technique). For coherence estimation and optimisation about 200 looks have been used, leading to very precise estimates of the coherence values and an unbiased optimisation. In Fig. 2 it can be observed that the runway area, consisting mainly of grassland, is more coherent in VV than in $\mathrm{HH}$, while the forested areas are more coherent in VV polarisation. The optimised coherence map obviously combines high coherent areas of both $\mathrm{HH}$ and VV polarisation and reaches in many areas coherences values, which are significantly higher than in the measured channels themselves. Tab. I gives an overview of the achieved mean coherence values over several selected areas of interest. It has to be noted that the improvements through coherence optimisation might appear small for the long-term interferometric pairs. Nevertheless, they are significant enough to turn many vegetated and strongly decorrelated areas into something analysable, particularly when comparing to the VV coherence.

From the fully polarimetric data, the 2 partial polarimetric modes HH-HV and VV-VH can be directly derived by eliminating one element of the scattering vector. The scattering 

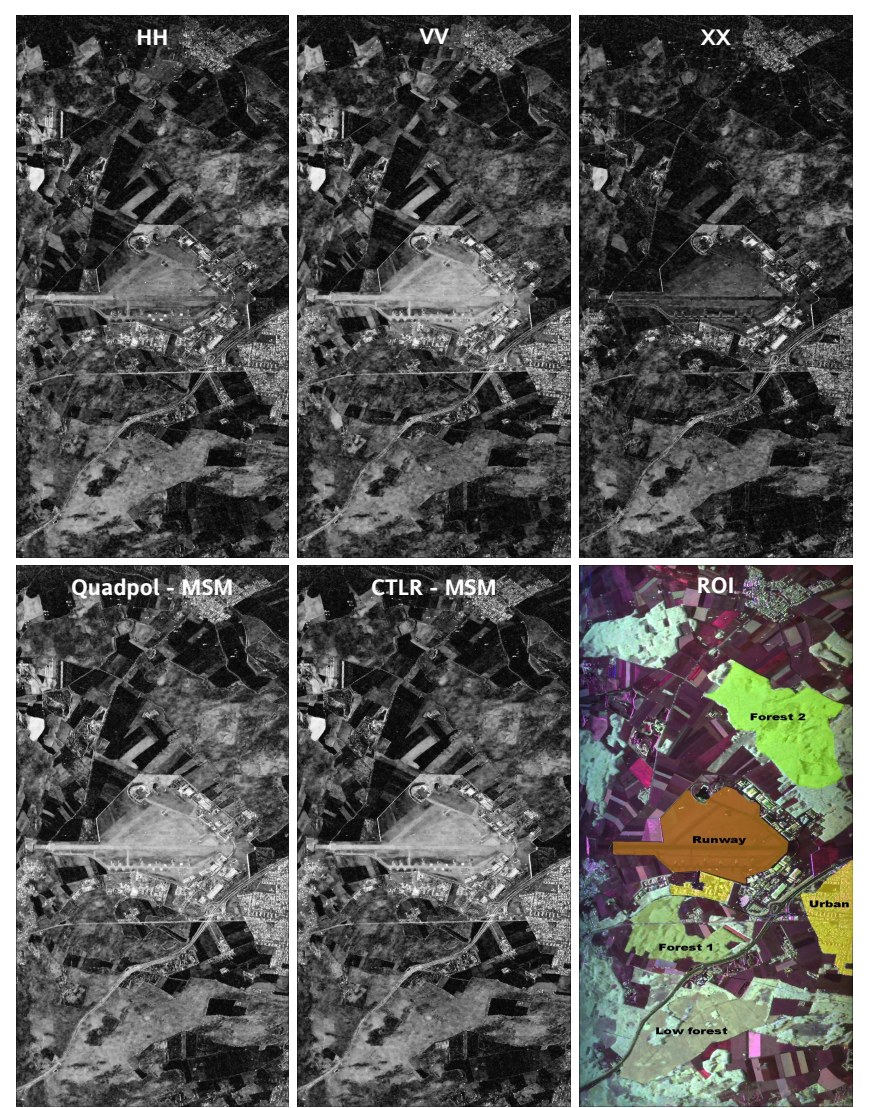

Fig. 1. Top row: Coherence maps of the test-site in the three channels directly measured by the sensor. Bottom row: Left: fully polarimetrically optimised coherence (MSM). Middle: Optimised coherence of the CTLR compact mode (MSM). Right: Amplitude SAR image overlaid with the areas of interest.

vectors of the 3 compact modes can be generated as follows:

$$
\begin{aligned}
\vec{k}_{\pi / 4} & =\left[S_{h h}+S_{h v}, S_{v v}+S_{h v}\right] / \sqrt{2} \\
\vec{k}_{D C P} & =\left[S_{h h}-S_{v v}+2 i S_{h v}, i S_{h h}+i S_{v v}\right] / 2 \\
\vec{k}_{C T L R} & =\left[S_{h h}+i S_{h v}, i S_{v v}+S_{h v}\right] / \sqrt{2}
\end{aligned}
$$

For each of the 5 dual-polarimetric modes, the $8 \times 8$ multibaseline coherency matrix is formed, filtered in the same way and optimised by the MSM and ESM algorithms. One of the resulting coherence maps (CTLR-MSM) can be found in Fig. 2, a summary of the mean coherence values for different areas of interest are given in Tab. II. The respectively best mode is marked in green, other modes, which reach a quite similar coherence level, are marked in light green.

Among the partial modes, VV-VH performs best for surfaces with low vegetation, while $\mathrm{HH}-\mathrm{HV}$ is clearly the better choice for all forested areas. In an urban environment, both partial modes perform similarly well. For the 3 compact modes, the situation is more complicated: In urban areas, the achieved coherence values are quite similar and best for the $\Pi / 4$ mode, although only slightly better than the ones of the partial modes. For the runway (low vegetation), the DCP mode seems to deliver in most cases the highest coherence among the compact modes, but it gets outperformed by the partial
VV-VH mode. For forested ares, again the $\Pi / 4$ mode seems to be the best compact mode, but the partial HH-HV mode is very close or even superior. Finally, for the low forest, CTLR is slightly better than its competitors, and significantly worse than the simple partial HH-HV mode. Again it has to be noted that the small differences between the mean coherence values are indeed significant and can make a strong difference when analysing decorrelated areas.

Comparing with the fully polarimetrically optimised case, one can observe that there is a significant loss in coherence of 5-20\% when only partial or compact modes are available. This holds particularly for areas with high vegetation.

Finally, Fig. 2 shows two deformation maps of the testsite, derived by DInSAR analysis of the data. Both maps are based on fully polarimetric ESM optimisation of the entire stack of 4 images. ESM has been chosen here to ensure that identical scattering processes are observed in the differential interferograms, i.e. to avoid a mixture of polarimetric and interferometric phase effects. Two interesting observations can be made: First, the coherence over forested areas is sufficient for DInSAR analysis even for a time span of one year. In fact, most agricultural areas are much less correlated, presumably due to changes in cultivation. Secondly, quite significant changes in the height of the phase centres can be observed over the forested areas. However, the growth of the trees is an unlikely factor for this, as the forest crown should be uncorrelated over such long time intervals. Additionally, the observed change is reverted for the second pair, therefore environmental factors (humidity, tree vitality, etc.) are more likely. For the moment, the reasoning for the observed height changes remain unclear and further investigations are required to clarify this effect.

\section{CONCLusions}

Coherence optimisation is a technique to enhance the interferometric coherence of fully polarimetric systems. This is achieved by the choice of a polarisation basis which has the highest possible coherence within the polarimetric observation space. The newly developed methods for the polarimetric optimisation of multi-baseline coherences provide the possibility of simultaneous constrained coherence optimisation for more than one baseline. These capabilities are particularly interesting in the area of differential interferometry and permanent scatterer analysis, where multiple data sets are involved.

When instead of a fully polarimetric system just a partial or compact polarimetric mode is available, only a reduced configuration space for the coherence optimisation is available. This results in coherences that are higher than in the monopolar case, but lower than for the fully polarimetric case. Among the recently discussed compact modes, the $\Pi / 4$ modes delivers the highest coherence over densly vegetated areas. However, the analyses presented in this paper reveal also that none of the compact modes have a significant advantage over the simpler partial modes in terms of coherence; in several cases they are even outperformed by them. Generally, the 
TABLE II

OPTIMISED COHERENCE VALUES FOR PARTIAL AND COMPACT POLARIMETRIC MODES. P1 = HH-HV, P2=VV-VH, C1 = CTLR, C2 = DCP, C3 = PI4

\begin{tabular}{|cc||cccc|cccccc|} 
ROI & Pair & P1-MSM & P1-ESM & P2-MSM & P2-ESM & C1-MSM & C1-ESM & C2-MSM & C2-ESM & C3-MSM & C3-ESM \\
\hline \hline \multirow{6}{*}{ runway } & $1-2$ & 0,826 & 0,816 & 0,898 & 0,896 & 0,901 & 0,892 & 0,901 & 0,889 & 0,883 & 0,870 \\
& $1-3$ & 0,388 & 0,376 & 0,560 & 0,552 & 0,547 & 0,536 & 0,544 & 0,531 & 0,552 & 0,540 \\
& $1-4$ & 0,319 & 0,309 & 0,433 & 0,425 & 0,423 & 0,412 & 0,429 & 0,418 & 0,430 & 0,418 \\
& $3-4$ & 0,422 & 0,420 & 0,573 & 0,568 & 0,553 & 0,547 & 0,573 & 0,568 & 0,567 & 0,561 \\
\hline \multirow{6}{*}{ urban } & $1-2$ & 0,708 & 0,685 & 0,713 & 0,691 & 0,718 & 0,686 & 0,714 & 0,683 & 0,717 & 0,683 \\
& $1-3$ & 0,457 & 0,422 & 0,450 & 0,412 & 0,468 & 0,433 & 0,468 & 0,432 & 0,472 & 0,437 \\
& $1-4$ & 0,453 & 0,428 & 0,455 & 0,430 & 0,464 & 0,436 & 0,463 & 0,435 & 0,468 & 0,439 \\
& $3-4$ & 0,517 & 0,506 & 0,517 & 0,504 & 0,528 & 0,512 & 0,531 & 0,514 & 0,536 & 0,519 \\
\hline \multirow{6}{*}{ forest 1 } & $1-2$ & 0,366 & 0,349 & 0,357 & 0,339 & 0,360 & 0,339 & 0,361 & 0,340 & 0,362 \\
& $1-3$ & 0,207 & 0,189 & 0,183 & 0,163 & 0,197 & 0,179 & 0,196 & 0,178 & 0,205 & 0,340 \\
& $1-4$ & 0,200 & 0,191 & 0,178 & 0,170 & 0,189 & 0,179 & 0,190 & 0,179 & 0,202 & 0,192 \\
& $3-4$ & 0,291 & 0,285 & 0,269 & 0,265 & 0,277 & 0,270 & 0,279 & 0,271 & 0,290 & 0,281 \\
\hline \multirow{6}{*}{ forest 2 } & $1-2$ & 0,418 & 0,403 & 0,387 & 0,365 & 0,401 & 0,374 & 0,400 & 0,373 & 0,401 & 0,371 \\
& $1-3$ & 0,259 & 0,243 & 0,205 & 0,186 & 0,243 & 0,225 & 0,245 & 0,226 & 0,260 & 0,242 \\
& $1-4$ & 0,228 & 0,220 & 0,193 & 0,183 & 0,214 & 0,203 & 0,213 & 0,201 & 0,232 & 0,219 \\
& $3-4$ & 0,348 & 0,343 & 0,299 & 0,297 & 0,329 & 0,320 & 0,331 & 0,323 & 0,351 & 0,342 \\
\hline \multirow{6}{*}{ low forest } & $1-2$ & 0,850 & 0,841 & 0,819 & 0,807 & 0,830 & 0,817 & 0,829 & 0,814 & 0,815 & 0,798 \\
& $1-3$ & 0,345 & 0,333 & 0,280 & 0,265 & 0,327 & 0,314 & 0,332 & 0,321 & 0,328 & 0,316 \\
& $1-4$ & 0,353 & 0,345 & 0,290 & 0,282 & 0,332 & 0,323 & 0,334 & 0,326 & 0,333 & 0,323 \\
& $3-4$ & 0,510 & 0,509 & 0,458 & 0,458 & 0,496 & 0,493 & 0,501 & 0,500 & 0,493 & 0,491 \\
\hline
\end{tabular}
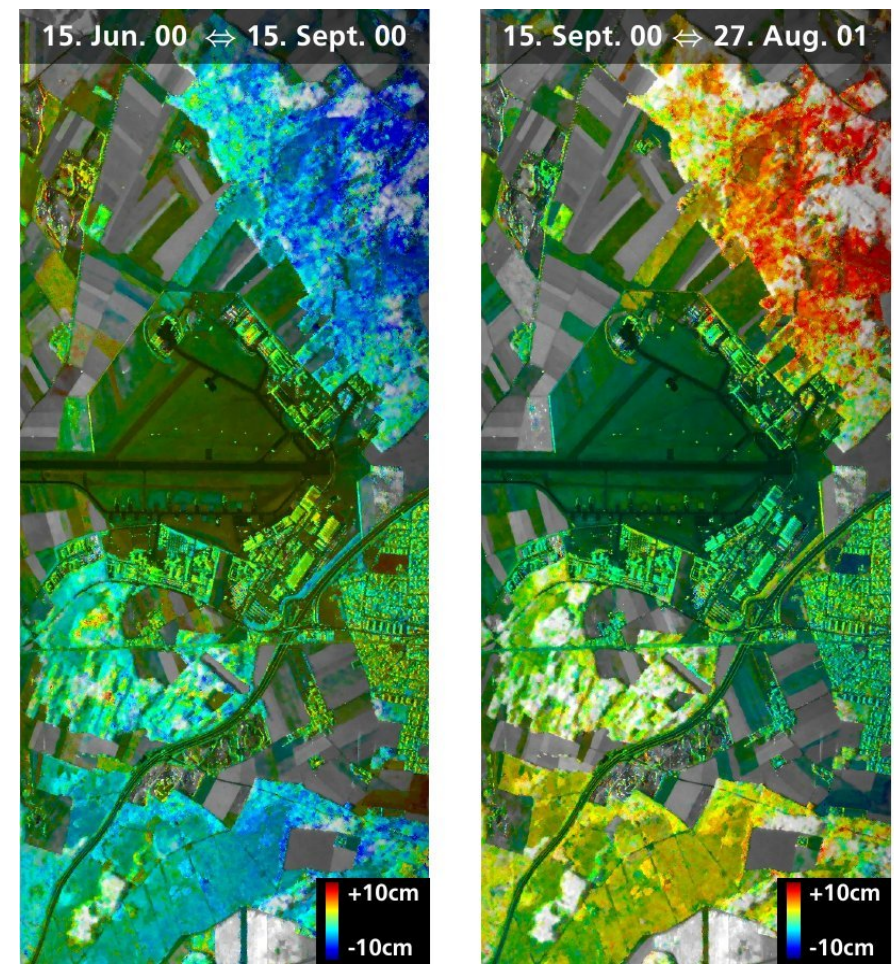

Fig. 2. DInSAR deformation maps for two time spans. Differential effects over some of the forested areas are clearly visible. Even over one year, sufficient correlation can be reached by coherence optimisation. optimisation potential of fully polarimetric PolInSAR data is significantly higher than the one of partial or compact modes.

Finally, for the first time deformation maps of forested areas have been presented, clearly showing changes in the mean backscattering height. This demonstrates that DInSAR analyses of forested areas are possible at longer wavelength in combination with polarimetric observations. The cause for the observed deformations is unclear and matter of further investigations.

\section{REFERENCES}

[1] R. Bamler and P. Hartl, "Synthetic aperture radar interferometry," Inverse Problems, vol. 14, pp. R1-R54, 1998.

[2] A. Reigber, M. Neumann, E. Erten, M. Jäger, and P. Prats, "Multi-baseline polarimetrically optimised phases and scattering mechanisms for insar applications," Proceedings of IGARSS'07, 2007.

[3] R. K. Raney, "Hybrid-polarity sar architecture," IEEE Transactions on Geoscience and Remote Sensing, vol. 45, no. 11, pp. 3397-3404, November 2007.

[4] J.-C. Souyris, P. Imbo, R. Fjortoft, S. Mingot, and J.-S. Lee, "Compact polarimetry based on symmetry properties of geophysical media: The pi/4 mode," IEEE Transactions on Geoscience and Remote Sensing, vol. 43, no. 3, pp. 634-646, March 2005.

[5] T. L. Ainsworth, M. Preiss, N. Stacy, M. Nord, and J.-S. Lee, "Analysis of compact polarimetric sar imaging modes," Proceedings of POLINSARO7, 2007. [Online]. Available: http://earth.esa.int/workshops/ polinsar2007/papers/84_ainsworth.pdf

[6] S. R. Cloude and K. P. Papthanassiou, "Polarimetric optimization in radar interferometry," Electronic Letters, vol. 33, no. 13, pp. 1176-1178, 1997.

[7] M. Neumann, L. Ferro-Famil, and A. Reigber, "Multibaseline polarimetric sar interferometry coherence optimization," IEEE Geoscience and Remote Sensing Letters, vol. 5, no. 1, pp. 93-97, January 2008.

[8] E. Colin, C. Titin-Schnaider, and W. Tabbara, "Investigation on different interferometric coherence optimization methods," Proceeding of POLINSAR'03, no. SP-529, April 2003. 